\title{
Selective spatial upregulation of intratumoral stromal aromatase in breast cancer patients: evidence for imbalance of local estrogen metabolism
}

\author{
Christian F Singer ${ }^{1,2}$, Anneliese Fink-Retter ${ }^{1}$, Daphne Gschwantler-Kaulich ${ }^{1}$, \\ Theresia Thalhammer $^{3}$, Gernot Hudelist ${ }^{1}$, Ruth Mueller ${ }^{1,2}$, Klaus Czerwenka ${ }^{4}$ \\ and Ernst Kubista ${ }^{1,2}$
}

\footnotetext{
${ }^{1}$ Division of Special Gynecology, University of Vienna Medical Center, Waehringer Guertel 18-20, 1090, Vienna, Austria ${ }^{2}$ Ludwig-Boltzmann-Institute of Clinical Experimental Oncology, ${ }^{3}$ Center for Physiology and Pathophysiology, ${ }^{4}$ Division of Gynecopathology, Medical University of Vienna, Vienna, Austria

(Requests for offprints should be addressed to C F Singer; Email: christian.singer@meduniwien.ac.at)
}

\begin{abstract}
The suppression of local estrogens levels is of key importance in the treatment of ER-positive breast cancer. Essentially all endocrine strategies act by either suppressing estrogen formation or competitively inhibiting receptor-binding in tumor cells. Nevertheless, little is still known about the local expression of aromatase and sulfotransferase which are the key modulators of intra-tumoral estrogen levels. We have performed immunohistochemostry to investigate the expression of aromatase and sulfotransferase in 42 samples obtained directly from malignant breast tumors, and compared it to biopsies obtained from uninvolved tissue in the vicinity of the invasion front, and to distant breast tissue. We found that aromatase was equally detectable in both tumor epithelial and stroma, but was mostly epithelial in non-malignant tissues $(P=0.00008$, Fisher's exact test). Also, aromatase protein expression was significantly more common in tumoral stroma when compared with peritumoral and distant breast stroma $(P=0.00005$, and $P<0.00001$ respectively). With the notable exception of cystosarcoma phylloides, sulfotransferase protein was detectable only in epithelial tissues, regardless of the location within the diseased breast. However, epithelial sulfotransferase was correlated with epithelial aromatase $(r=0.35461, P=$ 0.0009 , Spearman's $\rho$ test) and with the epithelial ER status $(r=0.29313, P=0.005)$. We have demonstrated a differential aromatase and sulfotransferase protein expression pattern that is dependent on the spatial relation to a malignant breast tumor. Our results indicate a net increase in intratumoral active estrogen levels through increased stromal aromatization, while physiological local inactivation by sulfotransferase activity remains essentially unchanged.
\end{abstract}

Endocrine-Related Cancer (2006) 13 1101-1107

\section{Introduction}

Breast cancer is by far the most common female malignancy in industrialized countries, and represents the leading cause of death among women between the ages of 30 and 45 years. Approximately, $60 \%$ of all breast cancer patients suffer from hormone-dependent breast cancer, which contains estrogen receptors (ER). While the possible biochemical role of estrogens in the development of breast cancer remains to be elucidated, estrogens directly promote growth and stimulate malignant behavior through the induction of local growth factors (Russo et al. 2003). In premenopausal women, the ovaries are the principle source of estradiol (E2), which functions as a systemically circulating hormone and acts on distal target tissues. However, in postmenopausal women, the ovaries have ceased to produce estrogen and extragonadal sites, such as fatty tissue, liver, bone, and smooth muscle cells become the major source of estrogen production. Here, estrogens are no longer solely an endocrine factor but appear to be 
produced mainly locally as paracrine factors. Some authors have even argued that systemic estrogens in postmenopausal women reflect a spill-over effect from locally acting hormones (Simpson 2003). While the local production in bone and adipocytes might serve physiological needs, malignant breast tumors also appear to produce their own estradiol locally (Sasano et al. 1997, Chetrite et al. 2000, Belanger et al. 2002). Intratumoral estrogen levels have been shown to be several folds higher than corresponding systemic levels (Pasqualini et al. 1995). Although malignant breast tissue contains all the enzymes necessary for the local biosynthesis of estrogens, there is increasing evidence that the cytochrome P450 enzyme aromatase is mainly responsible for the elevated local levels of biologically active estradiol. Aromatase catalyzes the conversion from C19 androgen precursors to C19 estrogens, and both its expression and activity are selectively upregulated in breast cancer tissues (Harada 1997, Miller et al. 1997, Santner et al. 1997). In addition, it has been reported that aromatase activity, when measured in vitro, is higher in breast tumors than in the fat next to the tumor or in normal breast fat (James et al. 1987).

While aromatase activity leads to an increase in local estrogen levels, the human estrogen sulfotransferase is responsible for the conversion of estrogens into their sulfated form, thereby reducing the levels of biologically active estrogen and counteracting the biological effects of aromatization. In analogy to the aromatase expression pattern, tumoral tissues have also been described to contain significantly higher levels of sulfotransferase activity when compared to normal breast tissue, and within malignant tumors, sulfatase levels are again higher in ER +/PR + tumors (Tseng et al. 1983). However, these observations are somewhat challenged by other groups who have demonstrated sulfotransferase expression in normal breast cells but not in breast cancer cells (Falany \& Falany 1996). Their results imply increased sulfotransferase activity in normal breast tissue, which in turn would result in decreased proliferation through decreased levels of biologically active estradiol. In tumor tissues, the lack of epithelial sulfotransferase activity could lead to higher levels of active estradiol and consequently increased tumor cell proliferation in estrogensensitive tumor cells (Pasqualini \& Chetrite 2005). While the hypothesis appears convincing, considerable debate still exists about the regulation of local estrogen levels in breast cancer.

We have therefore investigated the spatial expression pattern of epithelial and stromal aromatase and sulfotransferase protein expression by performing immunohistochemistry on tissues obtained directly from a malignant tumor, from non-malignant tissue in the vicinity of a tumor, and from uninvolved breast tissue obtained at a distance from the tumor.

\section{Materials and methods}

\section{Tissue specimen}

Tissue arrays containing 42 paraffin-embedded sets of tissues obtained directly from the tumor (tumor), from tissue less than $15 \mathrm{~mm}$ distant from the invasive front of a malignant tumor (adjacent), and from normal breast tissue (distant) were purchased from Biomax (Biomax Inc, Rockville, MD, USA). The dignity of the arrayed tissues and the presence of epithelial and stromal compartments was confirmed by an experienced pathologist (KC).

Breast cancer specimens $(n=5)$ used for the preparation of cytosolic extracts were obtained from patients operated for breast cancer at the Clinic for Obstetrics and Gynecology, AKH, Medical University Vienna. Informed consent was obtained from all patients, and the study was approved by the ethical committee of the institution.

\section{Immunohistochemistry}

Paraffin-embedded tissue sections were deparaffined and endogenous peroxidases were inactivated by a 15 min treatment with methanol containing $3 \%$ hydrogen peroxide. After a short rinsing step in tap water, non-specific binding was blocked with goat serum for $30 \mathrm{~min}$. The anti-aromatase polyclonal antibody was obtained from a rabbit immunoglobulin fraction of an antiserum prepared against human placental aromatase and was kindly provided by Dr N Harada, Fujita Health University Hospital of (Toyaoake, Japan) (Harada 1988). It was used at a dilution of $1: 1000$. The monoclonal mouse estrogen sulfotransferase Ab1 antibody (EST.1) was purchased from NeoMarkers (Fremont, CA, USA) and used at a concentration of $2 \mu \mathrm{g} / \mathrm{ml}$ with subsequent boiling of tissue sections in $10 \mathrm{mM}$ citrate buffer at $\mathrm{pH} 6.0$ for $15 \mathrm{~min}$.

$\mathrm{ER} \alpha$ was immunostained using the monoclonal antihuman ER $\alpha$ antibody 1D5 (NeoMarkers), diluted at a ratio of $1: 100$. All slides were incubated at $4{ }^{\circ} \mathrm{C}$ overnight, washed in PBS for $5 \mathrm{~min}$, and incubated with peroxidase-labeled dextran polymers conjugated to anti-mouse immunoglobulins (EnVision, DAKO Corporation, Carpinteria, CA, USA) for both sulfotransferase and aromatase. The primary ER $\alpha$ antibody was incubated with a biotinylated and horseradish peroxidase-labeled secondary antibody (Immuno Cruz Detection Kit, Santa Cruz Biotechnology, Santa Cruz, 
CA, USA). The sections were subsequently washed with PBS and subjected to diaminobenzidine for $10 \mathrm{~min}$. The reaction was finished by rinsing in distilled water for $5 \mathrm{~min}$. All slides were counterstained with Mayer's hematoxyline, dehydrated, and mounted with distyrene/tricresyl phosphate/xylene (DPX) mounting medium (FLUKA, Buchs, Switzerland). Human placental tissue served as positive controls for aromatase and liver tissue served as positive control for sulfotransferase protein expression.

The protein expression was semiquantified according to the immunoreactive score (IRS) developed by Remmele \& Schicketanz (1993). The IRS is the product of staining intensity (from 0 , no staining to 4 , strong staining) and percentage of positive cells ( 0 , $0 \%$ of cells stained; $1,<10 \%$ cells stained; $2,10-50 \%$ cells stained; $3,51-80 \%$ cells stained; $4,>80 \%$ of cells stained). The IRS can range from 0 to 12 and values of $>2$ are considered positive.

\section{Western blotting}

Tissues were homogenized in ice-cold lysis buffer (50 mM Tris- $\mathrm{HCl}, \mathrm{pH} 7.4,1 \mathrm{mM}$ EDTA, $2 \mathrm{mM}$ EGTA, $1 \mathrm{mM}$ dithiothreitol, $1 \mathrm{mM}$ phenylmethyl sulphonyl fluoride, $1 \%$ Nonidet NP-40, and combined phosphatase/protease-inhibitor cocktail (Sigma)). To remove cell fragments and nuclei, the crude homogenate was first centrifuged at $1500 \mathrm{~g}$ for $5 \mathrm{~min}$. After centrifugation of the supernatant for $1 \mathrm{~h}$ at $100000 \mathrm{~g}$, the cytosol was collected. For western blotting, $80 \mu \mathrm{g}$ protein was subjected to electrophoresis on $12 \%$ polyacrylamide gels. Separated proteins were blotted onto a polyvinylidene fluoride membrane (Pall Life Sciences, Ann Arbor, MI, USA) and probed with the monoclonal mouse estrogen sulfotransferase $\mathrm{Ab} 1$ antibody (final dilution 1:500) overnight at $4{ }^{\circ} \mathrm{C}$ and a peroxidase-conjugated secondary antibody (Sigma) for $2 \mathrm{~h}$ at room temperature. After treatment of membranes with the SuperSignalP chemiluminescent substrate (Pierce, Rockford, IL, USA), chemiluminscence signals were visualized on Kodak X-ray films. As a positive control, $2 \mu \mathrm{g}$ insect cell cytosols containing the baculovirus-expressed human estrogen sulfotransferase (SULT1E1) (Panvera Corporation, Madison, WI, USA) were used.

\section{Statistical analysis}

Fisher's exact was used to compare the qualitative expression pattern of aromatase and sulfotransferase in both the epithelial and stromal compartment in the three sets of breast tissues. Associations between ordinal parameters were evaluated by Spearman's $\rho$ test. For all analyses, a two-sided $P$-value $<0.05$ was considered statistically significant. SAS statistical software system (SAS Inc., Cary, NC, USA, version 8.1) was used for all calculations.

\section{Results}

\section{Spatial expression of aromatase and sulfotrans- ferase protein in intratumoral, peritumoral, and uninvolved breast tissue}

When the spatial expression pattern of aromatase and sulfotransferase was analyzed in tissue obtained from malignant tumors, microscopically uninvolved tissue adjacent to a malignant breast tumor, and healthy breast tissue from breast cancer patients, we found aromatase protein to be easily detectable in both stromal and epithelial cells, while sulfotransferase expression was usually confined to the epithelial compartment and was less pronounced (Table 1 and Fig. 1). While we did not observe differences between epithelial and stromal aromatase expression within malignant tumors $(P=0.43265$, Fisher's exact test $)$, epithelial expression of aromatase was significantly more common than stromal in peritumoral and normal tissues $(P=0.00008$ and $P<0.00001$ respectively, Fisher's exact test). No

Table 1 Immunohistochemical analysis of aromatase and sulfotransferase in human breast cancer

\begin{tabular}{|c|c|c|c|c|c|c|c|c|c|}
\hline & & \multicolumn{4}{|c|}{ Aromatase } & \multicolumn{4}{|c|}{ Sulfotransferase } \\
\hline & & 0 & + & ++ & +++ & 0 & + & ++ & +++ \\
\hline \multirow[t]{2}{*}{ Intratumoral } & Epithelial & 2 & 14 & 19 & 4 & 13 & 9 & 12 & 4 \\
\hline & Stromal & 5 & 24 & 10 & 2 & 37 & $1^{a}$ & 0 & 0 \\
\hline \multirow[t]{2}{*}{ Peritumoral } & Epithelial & 1 & 12 & 9 & 5 & 7 & 13 & 7 & 3 \\
\hline & Stromal & 19 & 19 & 1 & 0 & 34 & 0 & 0 & 0 \\
\hline \multirow[t]{2}{*}{ Normal } & Epithelial & 1 & 14 & 8 & 6 & 10 & 12 & 8 & 1 \\
\hline & Stromal & 32 & 7 & 0 & 0 & 33 & 0 & 0 & 0 \\
\hline
\end{tabular}

0 , no expression; + , weak expression; ++ , intermediate expression; +++ , strong expression as assessed by the immunoreactive score $\left(0-2={ }^{\prime} 0\right.$ '; $3-5={ }^{\prime}+$ '; $6-9={ }^{\prime}++^{\prime} ; 10-12={ }^{\prime}+++$ ').

${ }^{\mathrm{a} C}$ Cystosarcoma phylloides. 
A

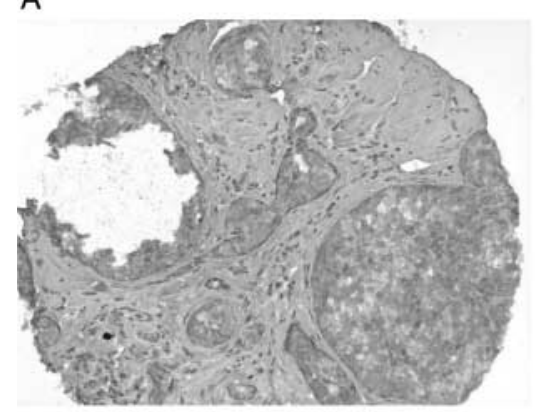

C

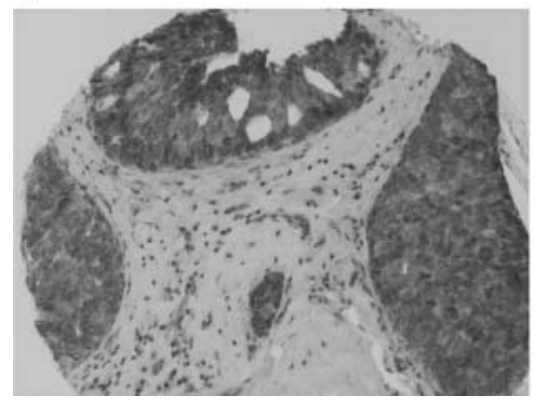

B

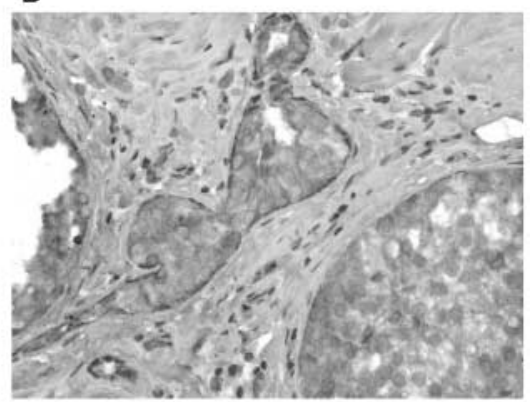

D

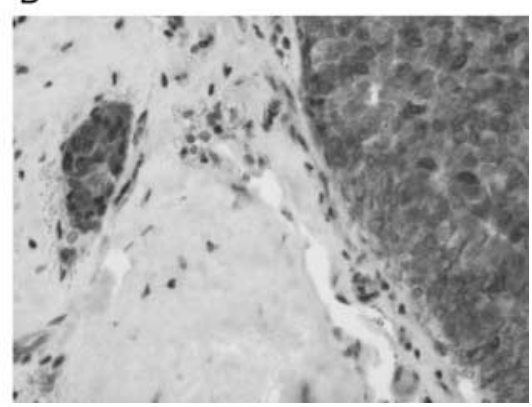

Figure 1 Aromatase and sulfotransferase protein expression in breast carcinomas. Brightfield photomicrographs show paraffinembedded tissue sections stained with diaminobenzidine (DAB) after immunohistochemical treatment with an anti-human aromatase antibody (A, B), an anti-human sulfotransferase antibody (C, D) as described in Materials and methods.

significant difference was found in the number of aromatase-expressing tissues when the epithelial components were compared within the three biopsy sets. However, stromal aromatase protein ion was significantly more common in tumoral tissues in comparison with peritumoral and normal tissues $(P=0.00005$, and $P<0.00001$ respectively, Fisher's exact test). In addition, peritumoral stroma exhibited aromatase significantly more often than stroma from distant breast biopsies ( $P=0.0039$, Fisher's exact test).

By contrast, sulfotransferase protein expression was exclusively confined to the epithelium, regardless of the location within the diseased breast. We observed weak stromal sulfotransferase expression only in the one case of a tumoral cystosarcoma phylloides biopsy that was included in the array. No significant difference in epithelial sulfotransferase protein was found when tumoral expression was compared with peritumoral $(P=0.42435$, Fisher's exact test) and with peripheral
$(P=1)$ tissue, and when peritumoral expression was compared with peripheral tissue $(P=0.56996)$.

Estrogen sulfotransferase expression was also demonstrated by western blotting in five cytosol samples prepared from human breast cancer specimens (Fig. 2). A specific immunoreactive band at approximately $34 \mathrm{kDa}$, which corresponds to the baculovirus-expressed estrogen sulfotransferase band in the insect cytosol indicates the presence of estrogen sulfotransferase.

\section{Correlation between aromatase, sulfotransfer- ase, and ER expression in normal, tumoral, and peritumoral tissue}

We then investigated possible associations between aromatase, sulfotransferase, and the ER status in both the epithelial and the stromal compartment (Table 2). Overall, the epithelial aromatase expression was significantly correlated with epithelial sulfotransferase

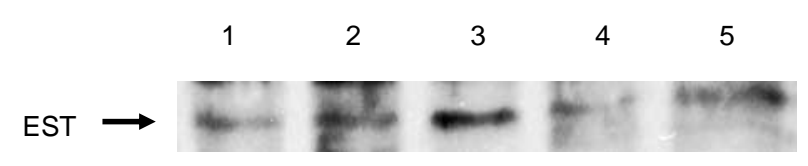

Figure 2 A representative western blot showing the estrogen sulfotransferase immunoreactive protein in cytosolic fractions $(80 \mu \mathrm{g}$ protein) prepared from five human breast specimens. 
Table 2 Associations between aromatase, sulfotransferase and ER protein expression in the epithelial and stromal compartment (Spearman's $\rho$ test)

\begin{tabular}{|c|c|c|c|c|c|c|}
\hline & $\begin{array}{c}\text { Aromatase } \\
\text { epithelial }\end{array}$ & $\begin{array}{c}\text { Aromatase } \\
\text { stromal }\end{array}$ & $\begin{array}{c}\text { Sulfotransferase } \\
\text { epithelial }\end{array}$ & $\begin{array}{c}\text { Sulfotransferase } \\
\text { stromal }\end{array}$ & ER epithelial & $\begin{array}{c}\text { ER } \\
\text { stromal }\end{array}$ \\
\hline Aromatase epithelial & $r=1.0000$ & $\begin{array}{l}r=1.6377 \\
P=0.1147\end{array}$ & $\begin{array}{l}r=0.35461 \\
P=0.0009\end{array}$ & $\begin{array}{l}r=0.08247 \\
P=0.4289\end{array}$ & $\begin{array}{l}r=0.17259 \\
P=0.1019\end{array}$ & n.a. \\
\hline Aromatase stromal & & $r=1.0000$ & $\begin{array}{l}r=0.0163 \\
P=0.5509\end{array}$ & $\begin{array}{l}r=0.05871 \\
P=0.5598\end{array}$ & $\begin{array}{c}r=-0.0029 \\
P=0.9774\end{array}$ & n.a. \\
\hline Sulfotransferase epithelial & & & $r=1.0000$ & $\begin{array}{l}r=0.10709 \\
P=0.2965\end{array}$ & $\begin{array}{c}r=0.29313 \\
P=0.0050\end{array}$ & n.a. \\
\hline Sulfotransferase stromal & & & & $r=1.0000$ & $\begin{array}{c}r=-0.0706 \\
P=0.5009\end{array}$ & n.a. \\
\hline $\begin{array}{l}\text { ER epithelial } \\
\text { ER stromal }\end{array}$ & & & & & $r=1.0000$ & $\begin{array}{l}\text { n.a. } \\
\text { n.a. }\end{array}$ \\
\hline
\end{tabular}

( $r=0.35461, P=0.0009$ Spearman's $\rho$ test), but not with stromal aromatase or sulfotransferase protein expression $(P=0.1147$, and n.s. respectively). Furthermore, no association was observed between epithelial aromatase and the ER status $(P=0.109)$. On the other hand, stromal aromatase protein expression was neither correlated with epithelial or stromal sulfotransferase, or with the epithelial ER status $(P=0.5509,0.5598$, and 0.9774 respectively). When the epithelial sulfotransferase protein expression was compared to the epithelial ER status, we found a significant positive correlation ( $r=0.29313, P=0.0050$, Spearman's $\rho$ test), but no such association was seen with stromal sulfotransferase expression $(P=0.2965)$. Stromal sulfotransferase was not correlated with any of the other two proteins, regardless of whether their expression was evaluated in the stroma or in the epithelium.

\section{Discussion}

Using immunohistochemistry, we have found a distinct pattern of aromatase expression that is dependent on the tissular relation to a malignant breast tumor. Our results are consistent with previous reports of increased levels of aromatase gene expression in the breast quadrants of breast cancer patients in which the malignant tumor was located (Simpson et al. 1994). However, in contrast to enzymatic assays, which are usually performed on whole-tissue homogenates, immunohistochemistry permits to identify the cellular source of aromatization. Over the last years, several antibodies have been used to detect aromatase, but most are associated with limitations that make interpretation of results difficult and have consequently lead to contradictory results (Lu et al. 1996). Only few antibodies, such as the monoclonal antibody \#677, which is suitable for the immunodetection of native aromatase, and the polyclonal antibody, which is suitable for paraffin-embedded tumor samples, have shown a good correlation to biochemical aromatase activity assays (Harada 1988, Sasano et al. 2005).

We have used the polyclonal antibody by Harada and detected selectively increased intratumoral stromal aromatase expression, which strongly indicates the existence of a paracrine regulatory mechanism via tumor cell-derived cytokines factors. This assumption is supported by the work of several groups who also detected increased aromatase expression in tumoral stromal spindle cells when compared to benign breast lesions (Santen et al. 1994, Sasano et al. 1994). While several cytokines are able to upregulate stromal aromatase protein and activity, Richards et al. (2002) have recently demonstrated that EGF and TGF $\beta$, which are both expressed by malignant breast epithelium, can directly upregulate aromatase activity in stromal cells in vitro. In addition, the two growth factors can also act indirectly by inducing COX-2 expression in stromal cells, which in turn could result in increased local PGE2 secretion and a further increase in stromal aromatase expression. However, there is also growing evidence for intrinsic epigenetic differences between tumor-associated and normal stroma; Chen et al. have proposed that physiological aromatase expression in breast fibroblasts is driven by the glucocorticoid-dependent promotor I.4, while the action of promotors I.3 and II are suppressed by the silencer negative regulatory element. In cancer cells and surrounding stroma, cAMP levels increase and aromatase promoters are switched to the cAMPdependent promoters I.3 and II (Chen et al. 1999). While the same group has also observed an upregulation in aromatase expression in breast cancer cells themselves through non-genomic action of ER $\alpha$ in conjunction with growth factor-mediated pathways in vitro, our finding of unchanged aromatase protein levels in both normal and malignant breast epithelium somewhat question the clinical relevance of their results 
(Chen et al. 2005). We have also been unable to detect a positive correlation between aromatase and ER status, which is in line with the observations by several other groups (Sasano et al. 1994, Pasqualini \& Chetrite 2005). However, our results somewhat contrast the findings of Esteban et al. (1992) who even detected a negative correlation between ER status and aromatase activity.

There are still considerable discrepancies concerning sulfotransferase activity in breast cancer. The exclusively epithelial sulfotransferase expression in our breast samples is consistent with previous scientific work where the enzyme was detected in both breast cancer cell lines and non-malignant SV40-immortalized breast epithelium (Anderson \& Howell 1995). However, others have been unable to detect sulfotransferase activity in most of the established breast cancer cell lines (Falany \& Falany 1996). Furthermore, while we did not find significant differences in protein expression between tumoral and non-tumoral tissue, one group has even described increased enzyme expression in normal breast epithelium when compared with malignant cells (Sharp et al. 1994). On the other hand, increased expression of both, estrogen sulfotransferase and aromatase was found in the tumor tissue as compared to the normal breast tissue, when samples were obtained from elderly women (Qian et al. 1998). Therefore, it is quite possible that hormonal changes, in particular, the lack of ovarian estrogen production during menopause, profoundly affect the expression of estrogen-metabolizing enzymes. The correlation between epithelial ER and epithelial sulfotransferase, as well as the correlation between epithelial sulfotransferase and epithelialaromatase indicates the presence of an auto- or paracrine-regulatory mechanism by which increases in local estrogen conversion and receptor expression are counterbalanced by increased estrogen sulfatation. Such a regulatory mechanism could be well involved in the regulation of tumor proliferation, since it has already been demonstrated that estrogen-dependent cells with high sulfotransferase expression grow more slowly than cells with low or undetectable levels of sulfotransferase (Qian et al. 1998). The induction of intratumoral sulfotransferase activity by low-dose progestins or the 19-nortestosterone derivative tibolone thus appears to be a plausible therapeutic strategy to lower local estrogen concentrations (Chetrite et al. 1998, 1999). However, the selective upregulation of aromatase expression in malignant tumors has already been demonstrated to result in a biologically relevant increase in local estrogen levels and significant stimulation of tumor cell proliferation, and therefore renders this enzyme a considerably more desirable target (Lu et al. 1996).
Indeed, the inhibition of intratumoral aromatase by highly selective inhibitors has recently been shown to be the most effective endocrine therapeutic strategy in postemenopausal receptor-positive women with breast cancer in several large clinical trials.

\section{Acknowledgements}

This study was supported by a grant from the Jubilaeumsfonds der Oesterreichischen Nationalbank, from Kommission Onkologie der Universitaet Wien, by a grant from Amgen, and by a grant from Medizinisch-Wissenschaftlicher Fonds des Buergermeisters der Bundeshauptstadt Wien. Supported by the Medizinisch-Wissenschaftlicher Fonds des Buergermeisters der Bundeshauptstadt Wien, by the Ludwig Boltzmann Society for Clinical Experimental Oncology, and by a grant from Amgen. The authors declare that there is no conflict of interest that would prejudice the impartiality of this scientific work.

\section{References}

Anderson E \& Howell A 1995 Oestrogen sulphotransferases in malignant and normal human breast tissue. EndocrineRelated Cancer 2 227-233.

Belanger C, Luu-The V, Dupont P \& Tchernof A 2002 Adipose tissue intracrinology: potential importance of local androgen/estrogen metabolism in the regulation of adiposity. Hormone and Metabolic Research 34 737-745.

Chen S, Zhou D, Okubo T, Kao YC \& Yang C 1999 Breast tumor aromatase: functional role and transcriptional regulation. Endocrine-Related Cancer 6 149-156.

Chen S, Ye J, Kijima I, Kinoshita Y \& Zhou D 2005 Positive and negative transcriptional regulation of aromatase expression in human breast cancer tissue. Journal of Steroid Biochemistry and Molecular Biology 95 17-23.

Chetrite G, Le Nestour E \& Pasqualini JR 1998 Human estrogen sulfotransferase (hEST1) activities and its mRNA in various breast cancer cell lines. Effect of the progestin, promegestone (R-5020). Journal of Steroid Biochemistry and Molecular Biology 66 295-302.

Chetrite GS, Kloosterboer HJ, Philippe JC \& Pasqualini JR 1999 Effect of Org OD14 (LIVIAL) and its metabolites on human estrogen sulphotransferase activity in the hormone-dependent MCF-7 and T-47D, and the hormoneindependent MDA-MB-231, breast cancer cell lines. Anticancer Research 19 269-275.

Chetrite GS, Cortes-Prieto J, Philippe JC, Wright F \& Pasqualini JR 2000 Comparison of estrogen concentrations, estrone sulfotransferase and aromatase activities in normal, and in cancerous, human breast tissues. Journal of Steroid Biochemistry and Molecular Biology 72 23-27. 
Esteban JM, Warsi Z, Haniu M, Hall P, Shively JE \& Chen S 1992 Detection of intratumoral aromatase in breast carcinomas. An immunohistological study with clinicopathological correlation. American Journal of Pathology 140 337-343.

Falany JL \& Falany CN 1996 Expression of cytosolic sulfotransferases in normal mammary epithelial cells and breast cancer cell lines. Cancer Research 56 1551-1555.

Harada N 1988 Novel properties of human placental aromatase as cytochrome P-450: purification and characterization of a unique form of aromatase. Journal of Biochemistry (Tokyo) 103 106-113.

Harada N 1997 Aberrant expression of aromatase in breast cancer tissues. Journal of Steroid Biochemistry and Molecular Biology 61 175-184.

James VH, McNeill JM, Lai LC, Newton CJ, Ghilchik MW \& Reed MJ 1987 Aromatase activity in normal breast and breast tumor tissues: in vivo and in vitro studies. Steroids 50 269-279.

Lu Q, Nakmura J, Savinov A, Yue W, Weisz J, Dabbs DJ, Wolz G \& Brodie A 1996 Expression of aromatase protein and messenger ribonucleic acid in tumor epithelial cells and evidence of functional significance of locally produced estrogen in human breast cancers. Endocrinology 137 3061-3068.

Miller WR, Mullen P, Sourdaine P, Watson C, Dixon JM \& Telford J 1997 Regulation of aromatase activity within the breast. Journal of Steroid Biochemistry and Molecular Biology 61 193-202.

Pasqualini JR \& Chetrite GS 2005 Recent insight on the control of enzymes involved in estrogen formation and transformation in human breast cancer. Journal of Steroid Biochemistry and Molecular Biology 93 221-236.

Pasqualini JR, Chetrite G, Nguyen BL, Maloche C, Delalonde L, Talbi M, Feinstein MC, Blacker C, Botella J \& Paris J 1995 Estrone sulfate-sulfatase and 17 beta-hydroxysteroid dehydrogenase activities: a hypothesis for their role in the evolution of human breast cancer from hormone-dependence to hormoneindependence. Journal of Steroid Biochemistry and Molecular Biology 53 407-412.

Qian Y, Deng C \& Song WC 1998 Expression of estrogen sulfotransferase in MCF-7 cells by cDNA transfection suppresses the estrogen response: potential role of the enzyme in regulating estrogen-dependent growth of breast epithelial cells. Journal of Pharmacology and Experimental Therapy 286 555-560.

Remmele W \& Schicketanz KH 1993 Immunohistochemical determination of estrogen and progesterone receptor content in human breast cancer. Computer-assisted image analysis (QIC score) vs. subjective grading (IRS).

Pathology, Research and Practice 189 862-866.

Richards JA, Petrel TA \& Brueggemeier RW 2002 Signaling pathways regulating aromatase and cyclooxygenases in normal and malignant breast cells. Journal of Steroid Biochemistry and Molecular Biology 80 203-212.

Russo J, Hasan Lareef M, Balogh G, Guo S \& Russo IH 2003 Estrogen and its metabolites are carcinogenic agents in human breast epithelial cells. Journal of Steroid Biochemistry and Molecular Biology 87 1-25.

Santen RJ, Martel J, Hoagland M, Naftolin F, Roa L, Harada N, Hafer L, Zaino R \& Santner SJ 1994 Stromal spindle cells contain aromatase in human breast tumors. Journal of Clinical Endocrinology and Metabolism 79 627-632.

Santner SJ, Pauley RJ, Tait L, Kaseta J \& Santen RJ 1997 Aromatase activity and expression in breast cancer and benign breast tissue stromal cells. Journal of Clinical Endocrinology and Metababolism 82 200-208.

Sasano H, Nagura H, Harada N, Goukon Y \& Kimura M 1994 Immunolocalization of aromatase and other steroidogenic enzymes in human breast disorders. Human Pathology 25 530-535.

Sasano H, Uzuki M, Sawai T, Nagura H, Matsunaga G \& Kashimoto O \& Harada N 1997 Aromatase in bone tissue. Journal of Bone and Mineral Research 12 1416-1423.

Sasano H, Anderson TJ, Silverberg SG, Santen RJ, Conway M, Edwards DP, Krause A, Bhatnagar AS, Evans DB \& Miller WR 2005 The validation of new aromatase monoclonal antibodies for immunohistochemistry - a correlation with biochemical activities in 46 cases of breast cancer. Journal of Steroid Biochemistry and Molecular Biology 95 35-39.

Sharp S, Anderson JM \& Coughtrie MW 1994 Immunohistochemical localisation of hydroxysteroid sulphotransferase in human breast carcinoma tissue: a preliminary study. European Journal of Cancer 30 1654-1659.

Simpson ER 2003 Sources of estrogen and their importance. Journal of Steroid Biochemistry and Molecular Biology 86 225-230.

Simpson ER, Mahendroo MS, Nichols JE \& Bulun SE 1994 Aromatase gene expression in adipose tissue: relationship to breast cancer. International Journal of Fertility and Menopausal Studies 39 75-83.

Tseng L, Mazella J, Lee LY \& Stone ML 1983 Estrogen sulfatase and estrogen sulfotransferase in human primary mammary carcinoma. Journal of Steriod Biochemistry 19 1413-1417. 